\title{
Tracking system based on accelerometry for users with restricted physical activity
}

\author{
L. M. Soria-Morillo†, J. A. Álvarez-García†, J. A. Ortegał, and \\ L. Gonzalez-Abril \\ $\uparrow$ Computer Languages and Systems Dept., University of Seville, \\ 41012, Seville, Spain \\ † Applied Economics I Dept., University of Seville, 41018, \\ Seville, Spain \\ \{lsoriamo, jaalvarez,jortega,luisgon\}@us.es
}

\begin{abstract}
This article aims to develop a minimally intrusive system of care and monitoring. Furthermore, the goal is to get a cheap, comfortable and, especially, efficient system which controls the physical activity carried out by the user. All this, is based on the data of accelerometry analysis which are obtained by a mobile phone.

Besides this, we will develop a comprehensive system for consulting the activity obtained in order to provide families and care staff an interface through which to observe the condition of the individual subject to monitoring.
\end{abstract}

\section{Introduction}

Just 30 minutes of moderate activity five days a week, can improve your health according to the Centers for Disease Control and Prevention ${ }^{1}$. By enabling activity monitoring at individual-scale, over extended period of time in a ubiquitous way, physical and psychological health and fitness could be improved. Furthermore, communication among relatives, friends or professionals could be enriched, showing graphics of weekly activity (very interesting for sportsman or elderly's relatives).

Current remote health monitoring applications are in an early commercial stage ${ }^{234}$ where application programmers, along with medical experts, are trying to analyze diverse parameters for providing wireless automated health care. In such a way, these systems need to transmit data to the backend very often, either by doctors' analysis, or due to computational intensive diagnosis algorithms that can't be executed efficiently on an embedded processor in a wearable device.

\footnotetext{
${ }^{1} \mathrm{http}: / / \mathrm{www} \cdot \mathrm{cdc} \cdot \mathrm{gov}$

2 http://www.fitbit.com

3 http://www.directlife.philips.com

4 http://www.miowatch.com
}

Commercial approaches use specific hardware, but we thought that modern mobile phones can achieve the same goals. However, high rates of physiological data have an adverse impact on the phone usability, not only due to expensive long-range communication, but also due to the costly data recovery and battery life.

With this paper, we hope to develop a dynamic, efficient and reliable system to control the user's monitored activities that have been practiced. Furthermore, the monitoring proposition must be as least intrusive as possible, since users, generally, are adverse to be controlled through traditional surveillance systems like surveillance cameras or sensors for all around their houses. A problem of these systems is the restricted sphere of action.

The system described above, tries to carry out the person's monitoring wherever he goes. This can be carried out thanks to that current mobile devices which incorporate accelerometry sensors, that means the user can carry the device at all times. This does that the range of our application will not be only the subject's house, work or training place, but it could be also controlled wherever he goes.

Our aim is to register every movement practiced by the user and classify it in different activities such as, for example, walking, running, jumping, going up and down stairs or even falls. Once having done that, the result of the classification will be visible by means of a web portal to user's family, doctors and anybody that the system administrator thinks it is necessary.

Furthermore, user's monitoring must carry out without its will be a too heavy load for owner subject. As we will see later, some existing devices allow make an user's monitoring, but the main problem is that only are available through proprietary hardware. Another problem happens as well, the user must wear an additional device; therefore, this can become uncomfortable and increase the risk of being forgotten by the user.

The opposite of above is that our system, on the user's side, expects to be integrated in the user's mobile 
device monitoring. The advantage of this decision it is just that mobiles are part of user's life style (every day more and more), and thus, the risk regarding loss or oversight is much lower than with an additional device. In addition to this, the boost and versatility of these devices make possible that the system possibilities can be increased.

The mobile devices connectivity is huge nowadays. In addition to the Wi-Fi technology that is more and more integrated in devices, the communication companies are done a strong vouch for $3 \mathrm{G}$ connectivity in these terminals. This technology allows us to connect users to Internet wherever they are. Thus, the access to Internet will be available any place and any time. Based on it, we can talk about developing an ubiquitous system that allows us to know subject's diary activity by means of information flowing through Internet.

\section{Related works}

The estimated physical activity, according to data obtained which are based on accelerometer, is, at the present, a research topic. There are some systems that are able to carry out this task. Among all of them, we will detail the most important and we will examine it to observe the difference and resemblance in accordance with our proposal.

The first difference we can observe among the developed systems up to now is the situation of the device and the device used for collecting information. There are systems that use proprietary hardware ([1], [2] and [9]), while others use general purpose hardware ([3], [4] and [10]), as the one we are describing. Obviously, to use generic hardware will result in a benefit, because the price and versatility of these devices are tricks to his advantage. However, these devices have a drawback: the limitations on the data collection quality. The accelerometers integrated on generic devices, such as the last generation of devices, have, overall, less quality in the data collection than other accelerometers that are integrated on specific devices. In this way, investigations that use specific hardware must focus on solving this trouble.

Other difference that we can find between the several researches is its own aim. In [5] we can see that the accelerometry sensor is located in a glove, which must wear the user and it is able to recognize several activities based on the hand movement. However, in other researches like [3], the sensor is situated in the user's pocket. Having arrived at this point, we can wonder what the most efficient method is. If we based on results, [5] and [8] has more precision than [3] and, furthermore, it is able to recognize larger number of activities. However, this solution could be uncomfortable for the user, thus the chosen system must be selected depending on the needed required.

In addition to the previous researches, other proposal for people with surveillance exists [7] that is not only based on accelerometry, but it also adds other elements like, for example, surveillance video cameras.

\section{System Design}

The remote surveillance system will be developed in three different environments: user's terminal, sever and remote query terminal. In the user's terminal will take place the data collection from the accelerometry sensor, data filtered a priori, classification of the user's activity and filtered a posteriori. The server environment will provide query interface for activities that the user is carrying out, communication service with the user's terminal and services needed for the communication with terminal query.

Finally, in query terminal will be executed the information query software. In design of system is contemplated that the software will be of two kinds: web interface or desktop interface. Web interface will be accessible to any user who desire look up the user's activity from any web browser by means of the application server. Desktop interface (both for Smartphone and for PC) will be communicated with the server through the web services offered by the own server.

In the next sections we will explain in more detail in what does consist the monitoring process and the activities control process. But before that, we will show a general view of the system. Figure [1] shows a flowchart of the system framework:

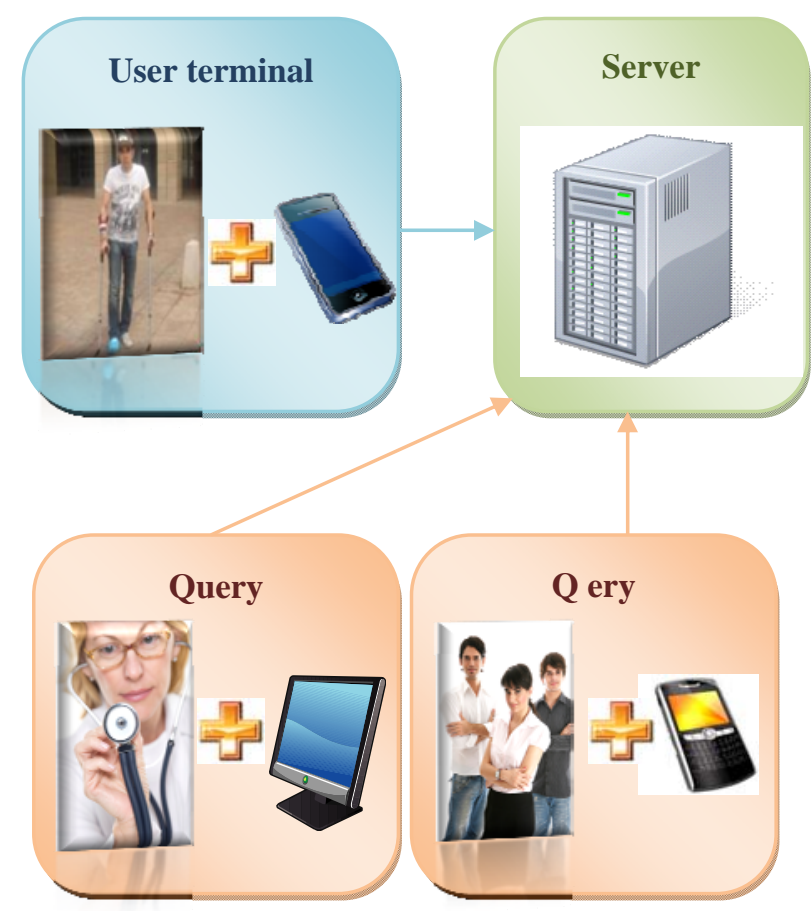

Figure 1: diagram of implementation 


\section{Obtaining and filtering data}

Following properties are desired for our activity recognizer installed on user device: manageable device, built-in GPS device, accelerometer sensor and its consumption should be enough for recharge the batteries each 24 hours at least.

Advances in microelectronics have reduced the cost of small and accurate sensors. As a result, accelerometers are embedded in modern mobile phones, the most ubiquitous device nowadays. Tri-axial accelerometers can measure G-force every axis and some features could be extracted to recognize the current user's activity.

Other profit of the activity monitoring system based on accelerometry is the low energy consumption produced in the device. This is essential when we talk about developing applications for mobile devices, because the bottleneck of these devices is the batteries. Furthermore, the user does not need recharge it constantly. Thus, the use of the system will be more comfortable for the user.

Specifically, we use the Samsung Omnia for our data collection, which contains a three axis accelerometer with a sensitivity of $+-2 \mathrm{G}$ and a resolution of $0.004 \mathrm{~g}$. Samsung device lasts more than 2 days with continuous use of this accelerometer, so the energy consumption will not be a problem and the user will not be constantly concerned by recharging his/her device.

The fact of having chosen the Omnia device for testing the system is because it integrates all the requirements described earlier: accelerometer, GPS device, operating system and all the facilities to work directly with all the data sensor of accelerometry. Furthermore, it is a current and, relatively, inexpensive device. This ensures us that the system is available for anyone without this meaning, a higher technology spending.

Although the data obtained are completely valid, it will be necessary to carry out a data filtering before making the classification. The filtering aim is to remove all the signal noise. In most of the cases, the noise of the accelerometry sensor is negligible, but exists a kind of noise that can affect seriously to the activity classification. This noise is produced by vibrations that take place on device when it is carried by the user.

We should consider an important aspect of our system: there is no restriction when it comes to choose the place where we must wear the device. This restriction is demanded by any related works. However, in our system, the user can wear the device where he wants. This increases the complexity of the development, because the mobile device subjection couldn't be peak condition. A bad subjection will produce unintended vibrations when the user carries out the activity. This is the reason of executing a filtering, in order to obtain valid data.

Throughout all the movement detection process, it has worked with the module of accelerometry. Usually, most accelerometer tends to measure accelerations that take place at three axis of device (triaxial accelerometer).
Figure [2] shows a graphic witch the three axis in a generic mobile device.

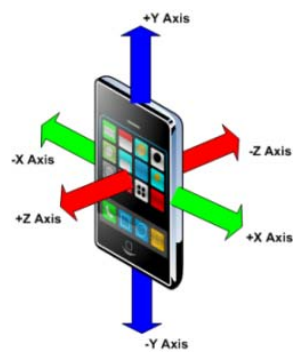

Figure 2: accelerometer 3-axis

\section{Definition 1:}

Accelerometry vector in time $t$, denoted as $\left|a_{t},\right|$ is defined as value that will be determined by next equality:

$$
\left|a_{t}\right|=\sqrt{a_{x}^{2} \cdot a_{y}^{2} \cdot a_{z}^{2}}
$$

, where $a_{x}, a_{y} y a_{z}$ are values of the acceleration in a certain time instant in the axis $x, y, z$ respectively.

To develop recognition of user activity accelerometry readings have been divided into temporal windows [Figure 3] then, analysis of a time window will result in a possible activity that the user is taking place during the time period that covers the window.

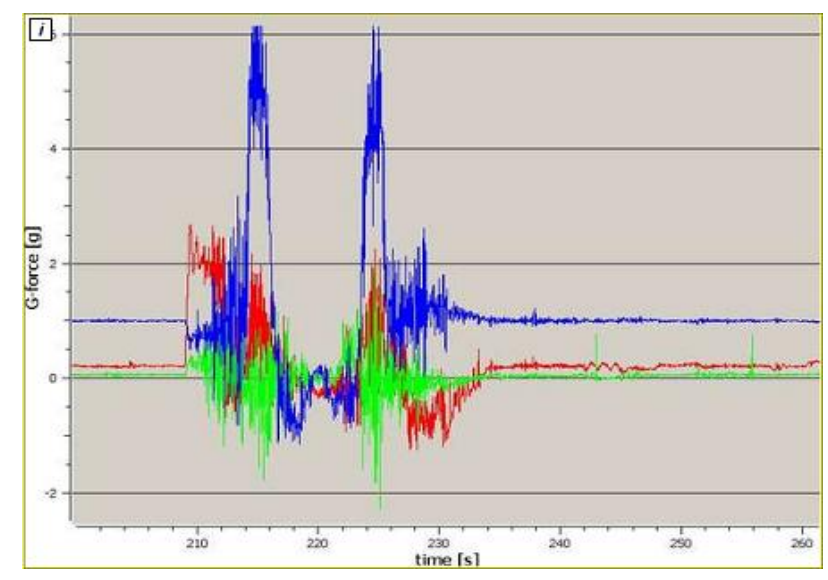

Figure 3: accelerometer signal into temporal window

Once recovered a window of 3 seconds of $\left|a_{t}\right|$, Fourier Transform is applied to get the frequency domain signal. Although the size of smaller window is desirable, the low frequency of accelerometer does not allow it (Samsung Omnia accelerometer only has $5 \mathrm{~Hz}$ ).

Cooley-Tukey algorithm was used to reduce the execution time $(O(N \cdot \log N)$. Then, Butterworth low pass filter was applied to eliminate noise caused by device vibrations. Furthermore, the frequency domain signal treatment allows us new readings, using interpolation, a very useful tool when the frequency is as 
low as our case. The latency of this transformation and filtering is around 2 seconds (in a separate thread) so our recognition activity system has a 5 seconds delay.

\section{Classification of the user's activity}

Since the entire process of classification (and training) will be made on the device itself, it is essential to minimize the computational cost. In addition, the design objective recognition of activity patterns is that all logic is done in the terminal. Since the learning until the classification, it should be based on stable pillars and the least heavy possible. This means that the recognition process must be, computationally, the least cost possible and as accurate as possible.

Although there are various techniques that can produce a more accurate of classification, are computationally much more expensive and further learning is, in most cases, unthinkable carry out in a mobile terminal.

The learning and classification method chosen is based on a probability table, and a second level, governed by a dynamic Markov chain which gives more control to the system. Prior to the detection process five vectors formed by six values must be identified.

\section{Definition 2:}

The rank vectors of the statistical variable $\beta$ is defined as one that sets up between its positions a range of values which can group readings values taken from a time window.

These vectors pretend to define a range for each of the statistical measures presented below:

- $\quad$ Arithmetic mean: $-\infty, 0.8,0.9,1.1,1.2$ and $+\infty$

- Range: $-\infty, 0.24,0.6,0.9,1.2$ and $+\infty$

- Variance: $-\infty, 0.09,0.15,0.25,0.35$ and $+\infty$

- Coefficient of Variation: - $\infty, 10,25,38,45$ and $+\infty$

- $\quad$ Minimum: $-\infty, 0.3,0.5,0.7,0.9$ and $+\infty$

That is, the vector corresponding to the arithmetic mean is composed of the above values that determine five ranges: $[-\infty, 0.8),[0.8,0.9),[0.9,1.1),[1.1,1.2)$, $[1.2,+\infty)$.

In learning, the user must perform each of the activities that can recognize the system. Unlike other works, the number and type of activities are not determined a priori, but the system administrator or users determine the activities that can be recognized. When performing the learning of a particular activity, each vectors corresponding to each of the statistical measure stores the number of temporal windows whose statistical measure is included in that range as well as the activity that is being developed. Thus, after completing the learning process, we have a series of vectors that contain at each position the number of temporal windows of each activity that have been detected in a certain range.

For determinate the activity that the user is carry out, the statistical values described above have been calculated based on the $\left|a_{t}\right|$ content in a given time window. After the expiry of the time corresponding to the time window, we have five values we use to determine the statistical activity that has taken place by the user during that time period. The next step is to visit the array of frequencies which are generated during the learning process. There will be a matrix for each activity and shall consist of $\mathrm{N}$ tables (where $\mathrm{N}$ is the number of activities that the system can recognize) composed by five rows (one for each statistical measure) and five columns (one for each range defined by the ranks vectors).

\section{Definition 3:}

The matrix of frequencies associated with $\partial$ activity is defined as that matrix which reflected in the position $[i, j]$ the number of readings that have been collected in the learning process of the activity $\partial$ of the statistical variable $i$ in range $j . J$ As the range defined in the vectors of range. After each process of learning, the values that made up the matrix are normalized to avoid dependence on the training time of each activity.

Having analyzed the current time window, we resort to the matrix learning $\partial$ of each activity. We will make a summation of those positions in the matrix in which the range of values of the statistical variable of row $i$, coincides with the value obtained from the time window. With this we get a number denoted as $\Omega_{t}(\partial)$.

\section{Definition 4:}

The Contest Sum, denoted by $\Omega_{t}(\partial)$, is defined as the contents sum of the matrix learning activity $\partial$ positions where the value obtained in the analysis of the time window $t$ for the statistical measurement $i$ coincides with the range defined in the standard position $j$.

Therefore, at this point, the probability of such activity is known based on reading conducted by accelerometry sensor and the study of the time window generated. It is also important bear in mind the reduced runtime of algorithm of detection. The algorithm used has linear complexity, so that its mobile terminal development is possible and would not entail excessive computational cost.

\section{Definition 5:}

Denoted by $\ddot{\partial}$ the most likely based activity $\Omega_{t}(\partial)$ obtained for each activity $\partial$ :

$$
\ddot{\partial}=\max _{\partial} \Omega_{t}(\partial)
$$

To clarify the process of classification of the user's activity, an example will be made below. First, the system collects accelerometry data every 200 milliseconds, although this frequency can be adjusted on the system configuration to allow lower frequency reading if the device support it. Every time the system get a data, its magnitude is calculated as it was detailed 
in Definition 1.This module will be stored in an array created for that purpose. This will happen during the time set for the time window, so after this period the vector of modules will be filled by all the readings taken. Then, from the stored data, statistical values listed in Definition 2 are calculated, i.e., Arithmetic Mean, Range, Variance, Coefficient of Variation and Minimum. For the present example, suppose that the values for these fields are:

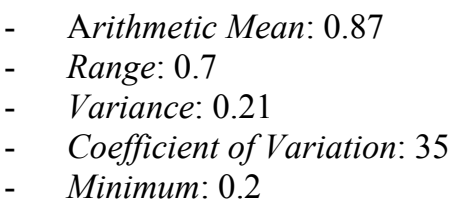

Now it's time to compare these values with the matrix described in Definition 3. To continue the previous example, assume that the system is able to recognize three activities: running, walking and jumping. In this way, our cube matrix shall consist of three two-dimensional arrays (one for each activity can recognize) $5 \times 5$ size (number of intervals for each statistical variable calculated and number of statistical variables respectively). Three-dimensional matrices resulting from the completion of the learning process are shown below:

\begin{tabular}{c|ccccc|} 
Running & $\begin{array}{c}\text { Interva } \\
1[1]\end{array}$ & $\begin{array}{c}\text { Interva } \\
1[2]\end{array}$ & $\begin{array}{c}\text { Interva } \\
1[3]\end{array}$ & $\begin{array}{c}\text { Interva } \\
1[4]\end{array}$ & $\begin{array}{c}\text { Interva } \\
1[5]\end{array}$ \\
\hline $\begin{array}{c}\text { Arithmetic } \\
\text { mean }\end{array}$ & 0 & $\mathbf{2 1}$ & 14 & 29 & 16 \\
Range & 0 & 0 & $\mathbf{1 7}$ & 30 & 33 \\
$\begin{array}{c}\text { Variance } \\
\text { Coefficien } \\
\text { t of }\end{array}$ & 0 & 10 & $\mathbf{2 0}$ & 35 & 15 \\
$\begin{array}{c}\text { Variation } \\
\text { Minimum }\end{array}$ & $\mathbf{5}$ & 20 & $\mathbf{1 0}$ & 14 & 0 \\
& & & & & \\
\hline
\end{tabular}

\begin{tabular}{c|ccccc|} 
Walking & $\begin{array}{c}\text { Interva } \\
1[1]\end{array}$ & $\begin{array}{c}\text { Interva } \\
1[2]\end{array}$ & $\begin{array}{c}\text { Interva } \\
1[3]\end{array}$ & $\begin{array}{c}\text { Interva } \\
1[4]\end{array}$ & $\begin{array}{c}\text { Interva } \\
1[5]\end{array}$ \\
\hline $\begin{array}{c}\text { Arithmetic } \\
\text { mean }\end{array}$ & 10 & 35 & 20 & 15 & 0 \\
Range & 10 & 15 & $\mathbf{2 7}$ & 18 & 10 \\
$\begin{array}{c}\text { Variance } \\
\text { Coefficien }\end{array}$ & 7 & 18 & $\mathbf{3 1}$ & 14 & 10 \\
$\begin{array}{c}\text { t of } \\
\text { Variation }\end{array}$ & & 10 & $\mathbf{3 7}$ & 19 & 9 \\
Minimum & $\mathbf{3 1}$ & 20 & 15 & 8 & 6 \\
$\begin{array}{c}\text { Jumping } \\
\text { Interval }\end{array}$ & Interval & Interval & Interval & Interval \\
\hline $\begin{array}{c}\text { Arithmetic } \\
\text { mean }\end{array}$ & 15 & $\mathbf{1 9}$ & 27 & 13 & 6 \\
Range & 18 & 21 & $\mathbf{3 0}$ & 11 & 0 \\
Variance & 10 & 16 & $\mathbf{2 6}$ & 15 & 13 \\
Coefficient & 3 & 24 & $\mathbf{2 7}$ & 18 & 7 \\
$\begin{array}{c}\text { of } \\
\text { Variation }\end{array}$ & & & & & \\
Minimum & $\mathbf{2 9}$ & 30 & 13 & 8 & 0
\end{tabular}

Once presented the learning matrix, based on Definition 4, the sum of learning cases for each of the activities that the system is able to recognize based on data obtained after analyzing the current time window are obtained. In the previous matrix, values in bold correspond to those intervals in which the group of values of the time window analyzed are contained. Based on the values obtained, the sums $\Omega_{t}(\partial)$ of each of the following activities will be:

$\begin{array}{ll}\text { - } & \text { Running: } \Omega_{t}(\text { Run })=21+17+20+10+5=73 \\ \text { - } & \text { Walking: } \Omega_{t}(\text { Walk })=35+27+31+37+31=\mathbf{1 6 1} \\ \text { - } & \text { Jumping: } \Omega_{t}(\text { Jump })=19+30+26+27+29=131\end{array}$

Once obtained the $\Omega_{t}(\partial)$ for each of the activities, based on Definition 5 , most likely activity $(\ddot{\partial})$ is chosen, whose sum $\Omega_{t}(\partial)$ has maximum value. For the present example, this activity is walking.

\section{Classify filter}

Most probable activity was recognized using accelerometer data, but the result of this classification suffers from noise and transitions between activities generate abrupt oscillations. A Markov chain will be introduced to control in a higher level the final classification of most probable activity.

The selection of a Markov chain to model the user's activity is based on the fact that if a user is doing an activity, there will be other related activities that he/she will do with a higher probability. For example, when a user is sitting, still sitting will be more probable than change to jumping activity.

Markov chain states will be the different activities that system could recognize. Given state $\partial_{i}$, probability of transition to state $\partial_{j}$ will determine transition probabilities of Markov chain $\left(p_{i j}\right)$. That is to say, change probability from one activity to other or the same every 3 seconds due to duration time window.

Obviously assigned probabilities to transition matrix are static, that is to say, they are defined by the system before beginning the activity recognition. However, dynamic probability index is proposed in this work:

\section{Definition 6:}

Dynamic probability index for transition between $\partial_{i}$ and $\partial_{j}$ will be denoted by $\alpha_{i j}$ and will be defined as the hope that activity $\partial_{j}$ is done on current state $\left(\Omega_{t}\left(\partial_{j}\right)\right)$ multiplied by the transition probability between $\partial_{i}$ and $\partial_{j}$ and normalized:

$$
\alpha_{i j}=\frac{\Omega_{t}\left(\partial_{j}\right) \cdot p_{i j}}{\sum_{j=1}^{n}\left(\Omega_{t}\left(\partial_{j}\right) \cdot p_{i j}\right)}
$$

From this moment, when we talk about transition probability of Markov chain from $\partial_{i}$ to $\partial_{j}$, we will refer to $\alpha_{i j}$. 


\section{Definition 7:}

Markov chain's transition matrix then will be denoted as T:

$$
T=\left[\begin{array}{cccc}
\alpha_{11} & \alpha_{12} & \ldots & \alpha_{1 j} \\
\alpha_{21} & \alpha_{22} & \ldots & \alpha_{2 j} \\
\vdots & \vdots & \ldots & \vdots \\
\alpha_{i 1} & \alpha_{12} & \ldots & \alpha_{i j}
\end{array}\right]
$$

We will define the state vector in instant $t$ and we will denote as $\alpha^{t}$ to the vector of length $j$, where $j$ is the number of activities that system can recognize and where each position of the vector corresponds to the probability that a transition to activity $j\left(\partial_{j}\right)$ in instant $t$ is done from activity done in instant $t-1$.

$$
\alpha^{t}=\left[\alpha_{j}^{t}\right]=\left[\sum_{i=1}^{n} \alpha_{j}^{t-1} \cdot \alpha_{i j}\right]=\alpha^{t-1} * T
$$

Once defined the state vector, we can select the most probable activity.

\section{Definition 8:}

We will define it as the current recognized activity and we denoted by $\hat{\partial}$ to the activity $\partial$ for which component $\alpha^{t}$ is larger:

$$
\hat{\partial}=\max _{j} \alpha_{j}^{t}
$$

It will be noted that the recognized activity after all the classification process is $\widehat{\partial}$, and it must not to match to $\ddot{\partial}$, due to the defined Markov chain includes additional information to the model.

To summarize, a very efficient Naïve classifier made an initial classification to then pass the output to a Markov chain that eliminates noise based on temporal knowledge of previous activity and the likelihood of transitioning into next activity.

\section{Server}

Once the classification of the activity has been completed and the system has recognized the activity, that the user is doing, it is time to send this information to the server. The aim is that the information was available to all users that have permission to look it. Thus, to control the activity of the user is possible.

This requires first to describe the architecture of the server in order to know the interactions available to it. The server will have a dual function. On one side will have a web services interface with which the system will be able to communicate with the user's mobile device monitoring, so that it is possible to send information about the activity which is being carried out and the time it began. On the other hand offer a web interface and a set of web services through which other users could view the information of activity that user is carrying out, either through a web browser or through special software that makes use of those services. Figure [4] shows the structure of the server.

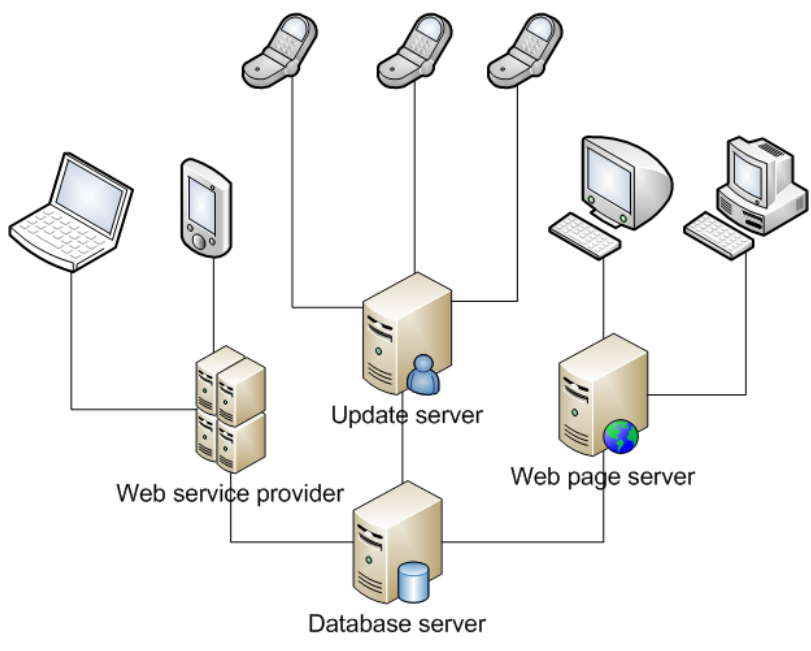

Figure 4: server diagram

The updated server will contain a set of web services that will allow the application, for recognizing the user's activity, can send the activity information to the server and store it in the database. For them will be defined at least one function: Updating activity. The parameters of this function are the time and date on which the user began the activity and the identifier of the activity itself with the ID of the user who performed it. This feature adds to the database a new entry with the data provided.

We must make an explanation before continue. The continuous transmission of information about the activity carried out could make the battery of the user mobile user expires quickly. Therefore, only new user activity will be sent on the servers when it change. That is, if an user left the device on the bedside table, for example, the last activity would be sent "without device", which would occur by detecting transmission that has left the device on a surface. Subsequently, it does not send any information to the server for the rest of the night, until the user returns to pick up his/her device to start the daily activity. In case that the device loses the connection with the server for any reason, for example the failure of network access, the activity performed by the user will be stored in the mobile device next to the time at which such activity occurred. As soon as the connection to the server is refreshed, such activities will be uploaded to the server to update the record of user's activities. With this process we increase the reliability of the system for consultancy work, as no false readings assigned activities due to failed connections to the server. First, the use of a keep alive signal was issued, but the idea was rejected in order to avoid overloading the server. Also, keeping alive signal would be meaningless if the above technique was used, because the data would be updated automatically once the server connection is restored.

This web service makes it possible to update the user's status, but not consulted. To this end, the web service provider and Web page server was developed. Both are connected to the database and obtain from it, the necessary information. Normally this 
information is the activity undertaken by a particular user during a certain time. In addition, it provides the ability to generate statistics about activities, duration and level of physical activity based on user activities detected.

\section{Experimental results}

Results about evaluation of the system are presented below. On one side will discuss the effectiveness and efficiency of the proposed architecture and secondly, the reliability and accuracy of the recognition of physical activity system proposed.

The activity of recognition based on the Naive-Bayes classifier with the noise reduction system based on the Cooley-Tukey and Butterworth filter has produced excellent results even with the presence of high noise levels.

To check the accuracy of the method, the system has been trained to be able to recognize 5 activities of daily life of any person: walking, running, jumping, going up and down stairs. The learning undertaken by all users subject under study has consisted of 15 minutes per activity, excluding jumping activity for which training has only been 5 minutes.

The following table lists the results of classification based on the number of temporal windows correctly and incorrectly recognized in the detection process:

\section{Activity Success Failure \% success}

\begin{tabular}{l|ccc|} 
Walking & 552 & 37 & $94.00 \%$ \\
Running & 258 & 18 & $93.00 \%$ \\
Stopped & 478 & 4 & $99.00 \%$ \\
Climb stairs & 331 & 25 & $93.00 \%$ \\
Down stairs & 441 & 41 & $92.00 \%$
\end{tabular}

Table 1: Activity recognition results

The results show that the prototype for detection of physical activity has got some very good results despite a short training. However, opposite to what one might think, a long learning can negatively affect the outcome of the classification, due to overlearning. This effect usually occurs when the number of training data is very high for certain activities while others too low. This problem causes that the less trained activities are more concrete when Naive-Bayes method described above makes the classification, while more trained activities have a higher probability in all ranks of classification determined by the rank vectors. Figure 5 shows a comparison between our systems under two conditions: normal learning (with similar training times for each activity) and overlearning with walking activity much more trained. On the $\mathrm{x}$-axis we can see the activities that the user has done during one hour. On the y-axis are shown the number of activities of each type recognized, this axis leads us to determinate the accuracy of recognition system. Finally, each color represents the activity recognized on an overlearned system, on a normal system and the true activity that user was doing.

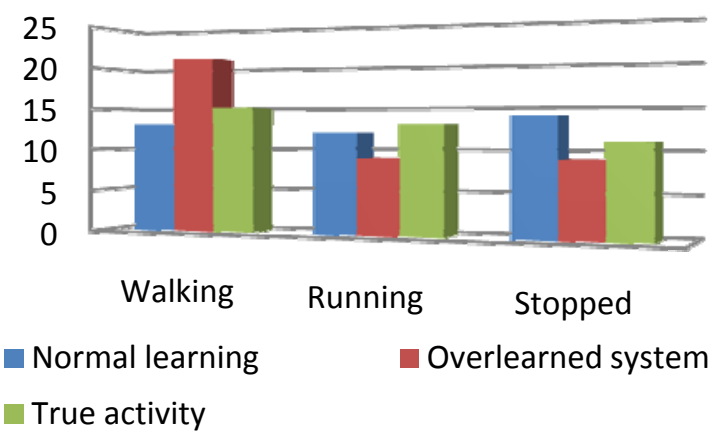

Figure 5: Overlearned system

\section{Conclusions}

We have achieved to perform a comprehensive system able to recognize, classify and share information about physical activity in a group of subjects. We have thus succeeded in controlling the activity of a user with a restriction of movement. This is very common in athletes who are in a period of restricted activity (e.g. rest) or elderly, since, thanks to the system, we can control anytime theirs activity. In other words, the activity that users are doing can be viewed by care persons without the need of being there, at the same place as user, allowing later the analysis of information of their activities.

Moreover, the entire system has been adapted to a goal: to be installed on mobile devices. This requirement has led to all methods of detection, classification and recognition of activities which must have a reduced computational cost. Due to the above, not only the risk of saturation of the mobile device system due to excessive calculations is reduced, but also power consumption is reduced to a minimum. This makes the user must recharge the device less frequently and therefore the system will be more functional and comfortable.

Finally, the proposed designed system has provided the capacity to access to the information of assisted subject through several ways. In this way, multitude of different devices (phones, PCs, PDAs, etc.) are covered by the system in order to access to the information anywhere and anytime.

\section{Acknowledge}

This research is partially supported by the MCI I+D projects FAMENET InCare (TSI2006-13390-C02-02) and ARTEMISA (TIN2009-14378-C02-01) and Andalusian Excellence I+D project CUBICO (TIC2141)

\section{References}


4 [1] Activity Monitoring System using Dynamic Time Warping for the Elderly and Disabled people. Sasiwan Paiyarom et al. $2^{\text {nd }}$ International Conference on Computer, Control and Communication. February 2009. ISBN: 978-160558-792-9

\& [2] Activity Recognition from Accelerometer Data. Nishkam Ravi et al. American Association for Artificial Intelligence, 2005. ISBN: 1-57735236-x

4 [3] Activity Recognition using Wearable Sensors for Elder Care. Yu-Jin Hong et al. IEEE Future Generation Communication and Networking, 2008. ISBN: 978-0-7695-3431-2

4 [4] Using acceleration measurements for activity recognition: An effective learning algorithm for constructing neural classifiers. Jhun-Ying Yang, Jeen-Shing Wang, Yen-Ping Chen. Pattern Recognition Letters, August 2008. ISSN: 01678655

* [5] Activity Recognition from Accelerometer Data on a Mobile Phone. Tomas Brezmes, JuanLuis Gorricho and Josep Cotrina. Lecture Notes in Computer Science, June 2009. ISBN: 978-3-64202480-1

* [6] Estimation of Activity Energy Expenditure: Accelerometer Approach. Jee Hyun et al. IEEE: Engineering in Medicine and Biology 27th Annual Conference. September 2005.

* [7] SmartBuckle: Human Activity Recognition using a 3-axis Accelerometer and a Wearable Camera. Yongwon Cho et al. In HealthNet '08: Proceedings of the 2nd International Workshop on Systems and Networking Support for Health Care and Assisted Living Environments. 2008, pp. 1-3. ISBN: 978-1-60558-199-6

* [8] Development ot a Single 3-Axis Accelerometer Sensor Based Wearable Gesture Recognition Band. Il-Yeon Cho et al. Springer Berlin: Lecture Notes in Computer Science. ISBN: 978-3-540-73548-9

* [9] Signal processing and machine learning for real-time classification of ergonomic posture with unobtrusive on-body sensors. Olsen G., Brilliant S., Primeaux D., and Najarian K. ICME International Conference on Complex Medical Engineering (CME 2009), pp.1-11, 9-11 April 2009.

* [10] Classification Technique of Human Motion Context based on Wireless Sensor Network. N. J. K. Joo Hyun Hong, Eun Jong Cha, Tae Soo Lee. Annual International Conference of the IEEE Engineering in Medicine and Biology Society. 2005 